\title{
Turbulent flow visualization by interferometric integral imaging and computed tomography
}

\author{
D.W. Watt \\ Dept. of Mechanical Engineering, University of New Hampshire, Durham, NH 03824, USA \\ C. M. Vest \\ Dept. of Mechanical Engineering, The University of Michigan, Ann Arbor, MI 48109, USA
}

\begin{abstract}
An experimental system using integral interferometric imaging and computer tomography for visualizing the structure of a turbulent, vertical helium jet is described. Integral images and tomographic integral data were obtained using a pulsed phase-shifted interferometer. The integral images revealed a sinuous overall jet structure and large-scale buckling motions in the far-field. Tomographic reconstruction of jet cross-sections at numerous axial locations were made for three turbulent jets at two different Reynols numbers, 2,800 and 4,300. Tomographic images revealed unmixed ambient fluid far inside the jet boundary and a bimodal concentration distribution. Image interpretation and experimental errors are discussed.
\end{abstract}

\section{Introduction}

Flow visualization has been an important tool in examining the structure of turbulent shear flows and there is an increasing emphasis on generating images of these flow's internal structure from multi-dimensional measurements of the scalar mixing field associated with them. The most common approach to obtaining these images is by the use of planar laser-induced fluorescence (LIF) or Rayleigh scattering techniques in which a laser sheet is passed through a given flow field, yielding a two-dimensional image of the flow structure (e.g. Dimotakis et al. 1983; Yip and Long 1986; Hiller and Hanson 1988).

Further understanding of the three-dimensional aspects of turbulent mixing fields requires development of threedimensional measurement techniques. One possible method for generating three-dimensional scalar measurements is optical tomography, which uses the measurements of optical path integrals of the real or imaginary part of the fluid refractive index to obtain cross-sectional point measurements. These measurements are recovered from the optical path measurements using an inversion process called tomographic reconstruction. Tomographic techniques require integral measurements from a range of viewing angle, and extensive computation time for the reconstruction process. Furthermore, their accuracy is limited by the amount of available data and depends on the complexity of the flow field. Despite these restrictions, tomographic methods have several potential advantages over planar techniques: (1) the integral measurements are fairly easy to obtain using absorption or interferometric methods; (2) they may be used to study flow geometries which are unamenable to planar techniques; and (3) improvements in image restoration techniques and computational speed may allow accurate measurements from incomplete data sets. Furthermore, the integral measurements themselves can be used to produce images which yield information similar to that of planar techniques about the internal flow structure; these images reveal variation in scalar concentration within the flow field in a similar way to diagnostic $x$-ray imaging of internal body structures.

In this paper a system for generating both integral images and tomographic reconstructions of a turbulent jet of helium is described. The integral measurements are made using pulsed, phase-shifting, holographic interferometry. The measured interferometric phase is used to generate integral images of the flow field, which show the large-scale topology of the jet's concentration field. Tomographic measurements were made by multi-angular viewing of the holographic interferograms. Cross-sectional tomographic reconstructions of the jet provide images of the concentration field at a series of locations along the jet axis. These images show the presence of unmixed, entrained ambient fluid well near the jet's center line.

\subsection{Pulsed phase-shifted interferometry}

Interferometric imaging is based on the phase variation of a spatially coherent optical wave traversing a flow field having a spatially inhomogeneous refractive index (Fig. 1). The spatial refractive index variation is caused by inhomogeneities in the fluid's density or chemical composition. The optical phase of the distorted wave is given by

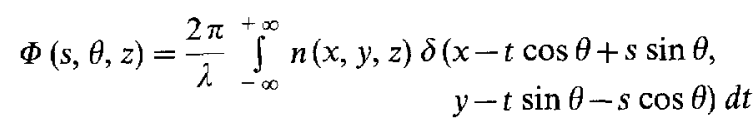




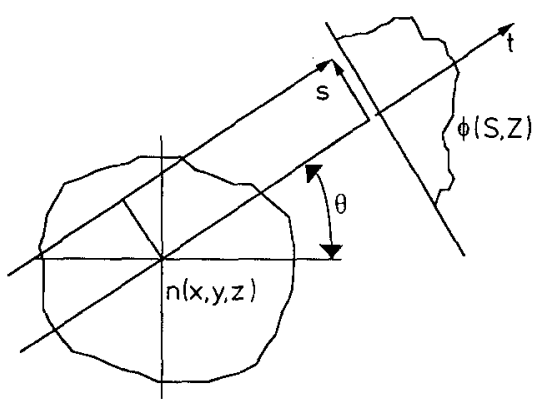

Fig. 1. Distortion of a plane wave by a refractive index field

where $\lambda$ is the optical wavelength, $n$ is the refractive index, $t$ is the pathwise coordinate, $\delta(x)$ is the Dirac delta function, $s$ is the transverse coordinate, $\theta$ is the viewing angle, and $z$ is an axial coordinate perpendicular to the direction of the probe wave.

Since the phase modulates the complex amplitude of the probe wave rather than its intensity, it must be recovered interferometrically. Interferograms encode the phase in a sinusoidal fringe pattern whose intensity (or irradiance) is given by (Vest 1979):

$I(x, y)=I_{0}(x, y)(1+\cos (\Phi(x, y)+\psi))$

where $I_{0}(x, y)$ is the background irradiance variation and $\psi$ is a uniform bias phase across the interferogram. If the interferometric phase variation is complex, the interferometric fringes are very convoluted and difficult to interpret visually. There are, however, several opto-electronic (e.g. Dändliker and Thalmann 1985), opto-digital (Dändliker and Thalmann 1985; Hariharan 1985), and fully digital (Kreiss 1986) techniques for recovering the interferometric phase. Watt and Vest (1987) have previously demonstrated phase-shifted interferometry for flow visualization using holographic interferometry in conjunction with optical phase shifting and computer image processing. By directly measuring the phase $\Phi(x, y)$ and displaying it as a gray-scale image, one can generate a true integral image of the scalar field which gives rise to the refractive index variations.

In this study, holographic interferograms of a turbulent flow field were recorded with a pulsed ruby laser $(\lambda=$ $694 \mathrm{~nm})$. A helium-neon laser $(\lambda=633 \mathrm{~nm})$ was used for hologram reconstruction and phase measurement. Partial compensation for the optical wavelength difference between the two lasers was achieved by the use of diffused illumination and closely spaced reference waves (Breuckmann and Thieme 1985). However, the diffuse illumination and wavelength change did cause some systematic errors due to holographic aberations [e.g. Meier (1965) and Leith et al. (1965)], overlapping holographic cross-reconstructions (Dändliker et al. 1982, 1976) and fringe localization (Vest 1979). The r.m.s. phase errors were found to be about 10 degrees; this level of accuracy was sufficient to demonstrate both integral and tomographic imaging. Because several sources of these errors can be eliminated by using different laser sources and illumination schemes, interferometric tomography is promising for adaptation to accurate tomographic measurement.

\subsection{Optical tomography: a review}

Tomographic reconstruction is the process of recovering the cross-sectional distribution of a source function (such as a two-dimensional refractive index distribution) from its integral projections. These projections are described by the Radon transform, $R(s, \theta)$, defined as:

$R(s, \theta)=\int_{-\infty}^{+\infty} f(x, y) \delta(x-t \cos \theta+s \sin \theta$,
$y-t \sin \theta-s \cos \theta) d t$.

This equation is the general form of Eq. (1). When the Radon transform is sampled over viewing angles from 0 to $\pi$, the source function $f$, can be obtained by direct transform inversion. This is generally implemented by first applying the backprojection operator (Deans 1983)

$Q(s, \theta)=\int_{-\infty}^{+\infty} \frac{1}{2 \pi(s-\alpha)} \frac{\partial}{\partial} \frac{R(s, \theta)}{s} d \alpha$,

separately to each Radon transform view. The source function is then reconstructed from $Q(s, \theta)$ in the following way:

$f(x, y)=\int_{0}^{\pi} Q(x \cos \theta+y \sin \theta, \theta) d \theta$

In many experimental geometries, the number of viewing angles is insufficient and the inversion formula cannot be directly applied. This missing-data problem is the subject of a large body of research and must be treated in some way virtually for every tomographic application. In the present study, a limited-data reconstruction scheme called consistent iterative convolution was used for reconstruction. This algorithm (Watt and Vest 1989) is a transform iteration technique based on numerically estimating the missing segments of the Radon transform, incorporating these estimates into the reconstruction using the direct inversion formula, and constraining the image using the available information. Image constraints include: (1) the flow region boundaries. (2) Non-negative helium concentration. (3) The source function's support, $S=\int_{-\infty}^{+\infty} \int_{-\infty}^{+\infty} f(x, y) d x d y$. The support quantity $S$ is the two-dimensional integral of the source function and can be found by numerically integrating $R(s, \theta)$ any individual Radon transform view with respect to $s$.

A variety of steady flows have been studied tomographically with both absorption and interferometric methods. These studies have treated the mixing fields of laminar jets (Bennet et al. 1984; Faris and Byer 1986) time-average turbulent jets (Santoro et al. 1981), pollution measurement (Byer and Shepp 1979; Stuck 1977), combustion diagnostics (Hertz 1985), natural convection (Sweeney and Vest 1973; Radulovich 1977) and transonic aerodynamics (Kittelson and Yu 1985). The only published study of unsteady flows 
measured tomographically appears to be the recent report by Snyder and Hesselink (1988) and Snyder (1988) of an elegant device for interferometric tomography of a helium jet. This study achieved the first truly three-dimensional tomographic scalar concentration measurement of turbulent flows.

\section{Integral and tomographic imaging of a turbulent helium jet}

To investigate the experimental application of integral imaging and interferometric tomography, a flow visualization study of the mixing field of round, vertical helium jet was carried out. Integral interferometric imaging was used to reveal the overall geometry of the flow and computed tomography was used to examine its internal topology. The helium jet was chosen as the test field object because: (1) it forms a compact phase object for convenient study and exhibits a range of dynamic behavor from laminar plume-like flow to momentum-driven turbulent jet-like flow; and (2) several studies of the instantaneous structure of similar flows exists for comparison.

\subsection{Test section and flow facility}

The test facility (Figs. 2 and 3 ) consisted of a flow field apparatus for recording the holograms and a separate device for reconstructing the holograms and processing the interferometric phase. The flow field apparatus (Fig. 2) exhausts the jet through a rectangular enclosure. Mounted on the enclosure are two scatter plates which diffusely illuminate the flow field. Opposite the scatter plates are perpendicular holographic plates, which are illuminated by a single collimated reference wave. In this way, one reflection hologram and one transmission hologram can be recorded simultaneously. The use of a single reference wave greatly simplifies the alignment of the digital interferometer during reconstruction, allows for efficient utilization of the available laser energy and it minimizes the number of necessary optical elements. The interferograms are recorded using the tilting reference wave method described by Breuckmann and Thieme (1985) for pulsed interferograms.

The developed holograms were mounted in the reconstruction device (Fig. 3), illuminated with a projection Michelson interferometer to facilitate phase-shifting (Breuckmann and Thieme 1985), and viewed with a railmounted CCD array camera attached to a rotary machine table. By centering its rotational axis in the holographic image of the jet, the object could be viewed from a range of directions while maintaining a known frame of reference for the image coordinates. The two holograms were aligned to eliminate image offset and minimize holographic magnification and aberration. This is achieved when $\tan \theta_{r}=\mu \tan \theta_{c}$, where $\theta_{r}$ and $\theta_{c}$ are the angles of the reference and object waves with the hologram surface normal, and $\mu$ is the ratio of the recording and reconstruction wavelengths.

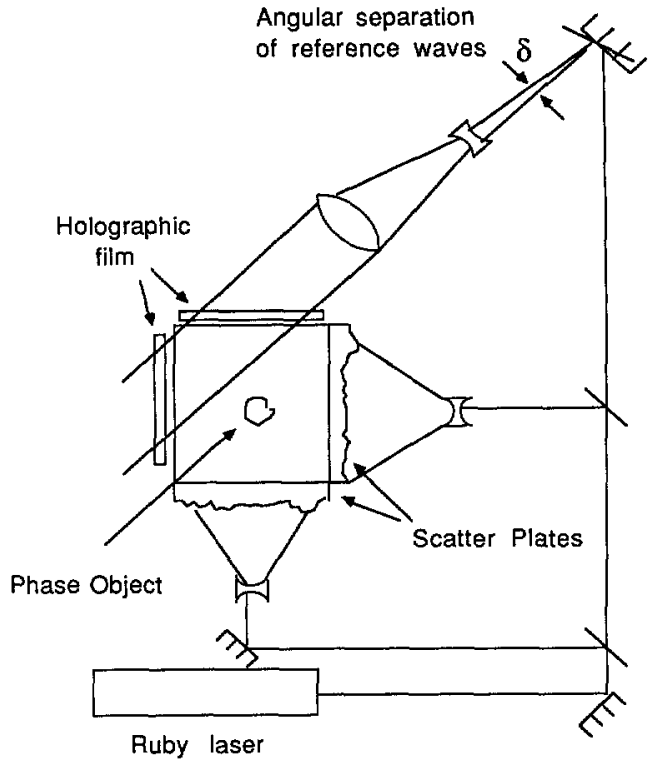

Fig. 2. Recording geometry for interferometric tomography

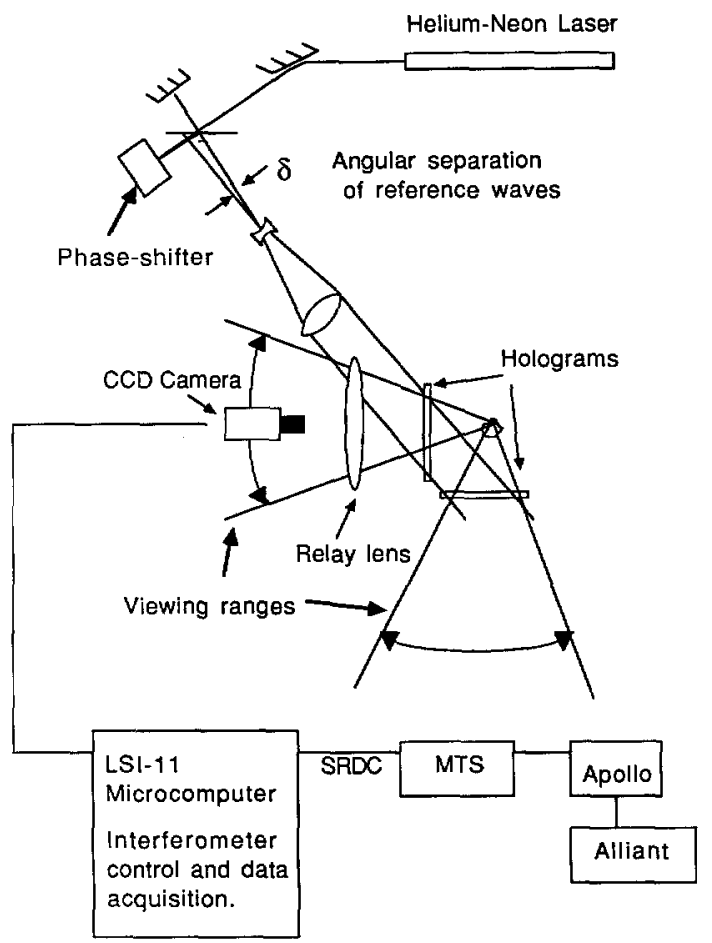

Fig. 3. Reconstruction geometry for interferometric tomography

The jet used in this study was generated by exhausting a steady flow of helium from a vertical pipe orifice which was $2.35 \mathrm{~mm}(3 / 32$ of an inch) in diameter. The pipe was 85 diameters long and the flow rate was controlled by a Flowrator valve. The pipe Reynolds numbers for the flows used in this study varied from 250 to 1,250. Application of a formula suggested by White (1974) for the entrance length of 

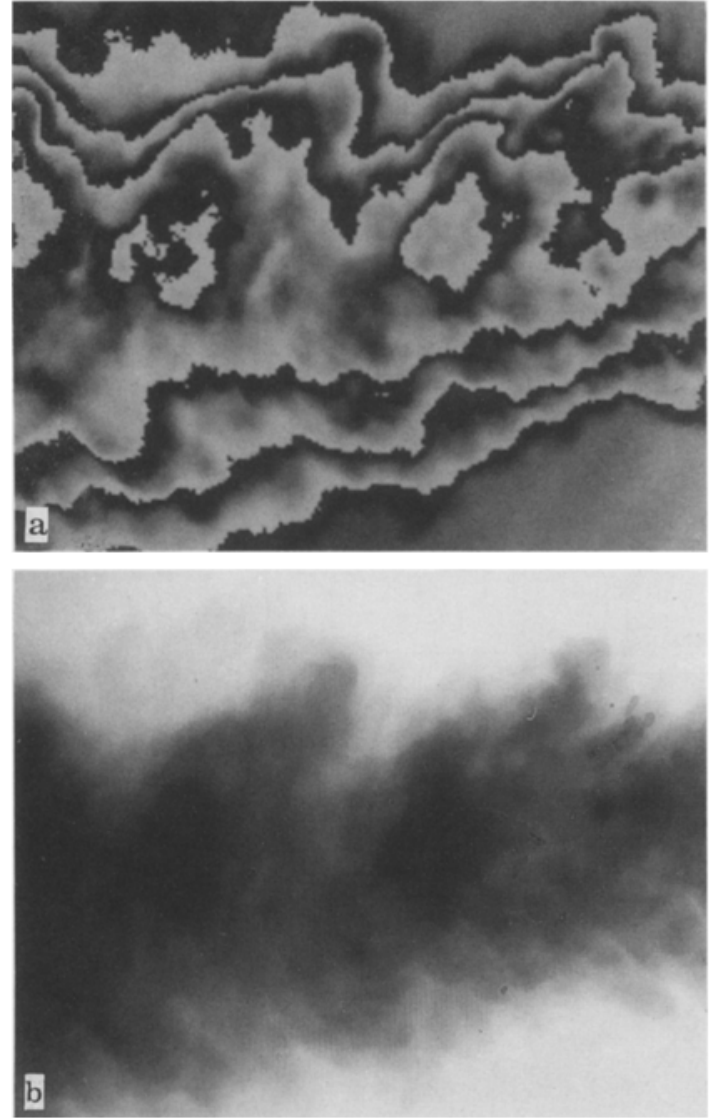

Fig. 4. a Data and $\mathbf{b}$ integral images of turbulent helium jet

laminar pipe flow indicates that these pipe flows are fully developed or, in the $R e=1,250$ case, nearly so. We therefore can assume that the initial jet velocity profiles are parabolic and use this velocity profile to compute the inlet momentum.

\subsection{Image acquisition and tomographic reconstruction}

The image acquisition system used in this study consists of a Cohu 4800 CCD array camera combined with a Datacube QVG/AFA image storage board driven by an LSI-11 based computer. The images are made up of a $374 \times 244$ element $\times 8$-bit pixel array. The phase-shifted data images were recorded and then smoothed with a nearest-neighbor averaging filter. The interferometric phase computations were then performed on the host computer.

A sample data image and its corresponding integral image for a turbulent jet are shown in Fig. 4. The data images are grey scale displays of the interferometric phase modulo $2 \pi$. The integral images were generated by accounting for the fringe orders to determine the absolute phase, normalizing these phase values to eight bits, and displaying them as a grey scale image. These images are used to obtain the following tomographic data: (1) the values and coordinates of the measured integrals, (2) the constraint radius, and (3) the support value, $S$. The Radon transform coordinates, $s$ and $\theta$ are obtained by pixel counting in the data image and by measuring the rotation of the machine table, respectively. The constraint radius (to apply constraint no.1) is taken to be 1.2 times local visible radius. In the case of the turbulent jet, the visible width and, consequently, the constraint radius, increase in the downstream direction with a half-angle of about $11^{\circ}$. The increase in jet width necessarily increases the radius of the tomographic support region, thereby increasing the number of pixels per view required for tomographic reconstruction from 45 near the pipe outlet to 205 at the farthest downstream point. The support value, $S$, is simply the sum of the Radon transform measurements for a single view and, in the turbulent cases, had standard deviations of $3 \%-8 \%$. The support constraint used the mean support value. Tomographic data were transferred to various larger-scale computers for processing. Reconstruction was very computationally intensive because of the numerous iterations required by the limited-data algorithm. In one extreme example, the reconstruction of a single $205 \times 205$ pixel image required $15 \mathrm{~h}$ of CPU time on an Apollo DN460. Although this time requirement was partially dependent on the relatively small range of viewing angles in these experiments, excess computation time is an endemic problem for many practical tomographic geometries. These problems may be partially alleviated by parallel computing. This was demonstrated rather dramatically when the previous example was run on an Alliant F/X 8 parallel-vector machine (with the algorithm adapted for parallel processing) in 20 min of terminal time, representing a roughly thirty-fold improvement.

\subsection{Physical meaning of integral and tomographic data}

The information contained in the integral images can be derived from the definition of the interferometric phase and the Gladstone-Dale relation. The interferometric phase $\Phi(s, \theta, z)$ given by Eq. (1) represents the Radon transform $R(s, \theta)$ [Eq. (2)] of a refractive index field $\Delta n(x, y, z)$, where $\Delta n$ is the refractive index change between the two holographic exposures. The refractive index, $n$, of a gas mixture is given by (Vest 1979):

$n-1=\sum_{i} K_{i} Y_{i} \varrho_{i}$

where $K_{i}$ is the Gladstone-Dale constant of the constituent $i, Y_{i}$ is its mole fraction and $\varrho_{i}$ is its density at the ambient pressure. The refractive index change between a field containing only air and one containing a helium-air mixture is given by:

$\Delta n=\left(K_{\mathrm{air}} \varrho_{\mathrm{air}}-K_{\mathrm{He}} \varrho_{\mathrm{He}}\right) Y_{\mathrm{He}}=\Delta(K \varrho) Y_{\mathrm{He}}$.

The Radon transform measurements, therefore, represent integral projections of the distributed mole fraction $Y_{\mathrm{Hc}}$ (multiplied by a scalar constant). The point values obtained by the tomographic reconstruction are, therefore, proportional to the local mole fraction. The mass of helium contained at any point is the product of the local helium mole 
fraction, helium density $\varrho_{\mathrm{He}}$, and the volume of the sampling element.

The support term $S(z)$, computed from these data is given by:

$S(z)=\frac{2 \pi}{\lambda_{r}} \Delta(K \varrho) \iint_{-\infty}^{+\infty} Y_{\mathrm{He}}(x, y) d x d y$

where $\lambda_{r}$ is the recording wavelength. The support is related to $M(z)$, the total mass of helium contained in a section of width $d z$ at an axial location $z$ by:

$M(z)=\frac{\varrho_{\mathrm{He}}}{\left(2 \pi / \lambda_{r}\right) \Delta K \varrho} S(z) d z$

\section{Integral imaging of a turbulent helium jet}

The dynamics of a turbulent, buoyant jet are governed by its initial momentum flux $J_{0}$, its buoyancy flux $B$, its initial velocity distribution, and the density of the jet and the ambient fluids. In the far field these flows are assumed to become self-similar and are described as momentum-dominated (jetlike), transitional or buoyancy-dominated (plume-like), depending on the distance downstream. The dimensionless numbers at the inlet that characterize these flows are the densimetric Froude number $F$, and the inlet Reynolds number $R e_{0}$, defined as:

$F=\frac{J_{0}}{g \Delta \varrho \pi d^{3}} \quad$ and $\quad R e_{0}=\left(\frac{2}{\sqrt{\pi}}\right) \frac{\left(J_{0} / \varrho_{\infty}\right)^{1 / 2}}{v_{\infty}}$.

Integral images of a vertical helium jet into air with range of Froude numbers from 160 to 37,500 are displayed in Figs. 5-7 (the upward direction is from right to left). At low Froude numbers the flow stays laminar throughout the visualization field. As the Froude number increases, the flow becomes unstable in the far field of the flow, with the onset of instability proceeding upstream with increasing Froude number. Finally, a turbulent, jet-like flow issues from the pipe. These integral images can be used to assess qualitatively the instantaneous structure of the flow field.

In their review of experimental data on buoyant jets, Chen and Rodi (1980) developed the following criteria for the jet-like, transition, and plume-like regimes:

$$
\begin{aligned}
F^{-1 / 2}\left(\frac{\varrho_{0}}{\varrho_{\infty}}\right)^{-1 / 2}\left(\frac{z}{d}\right)<0.5 & \text { Jet-like } \\
0.5<F^{-1 / 2}\left(\frac{\varrho_{0}}{\varrho_{\infty}}\right)^{-1 / 2}\left(\frac{z}{d}\right)<0.5 & \text { Transition } \\
F^{-1 / 2}\left(\frac{\varrho_{0}}{\varrho_{\infty}}\right)^{-1 / 2}\left(\frac{z}{d}\right)>5 & \text { Plume-like }
\end{aligned}
$$

where $z$ is axial coordinate and $d$ is the pipe diameter. For the fully-turbulent examples considered in this study, the Froude numbers ranged from 3,800 to 37,300 , which resulted
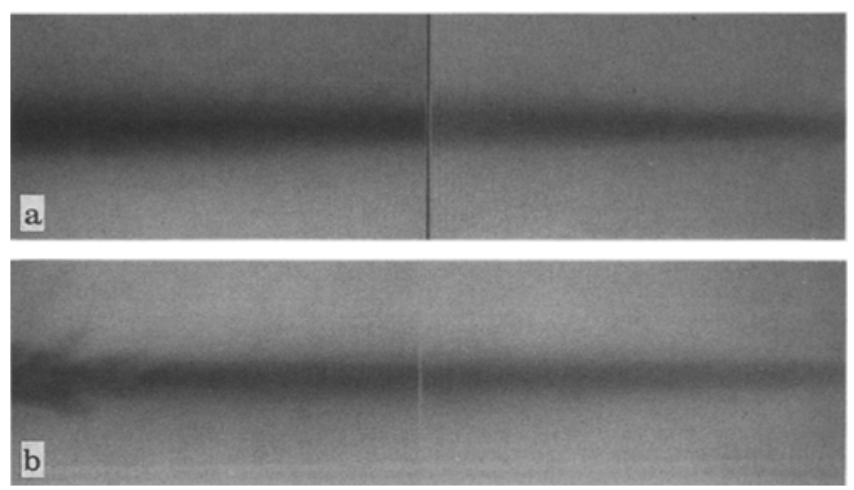

Fig. $5 \mathbf{a}$ and b. Integral visualizations of helium jets: a $F=405$, $=445 ; b=1,579,=850$
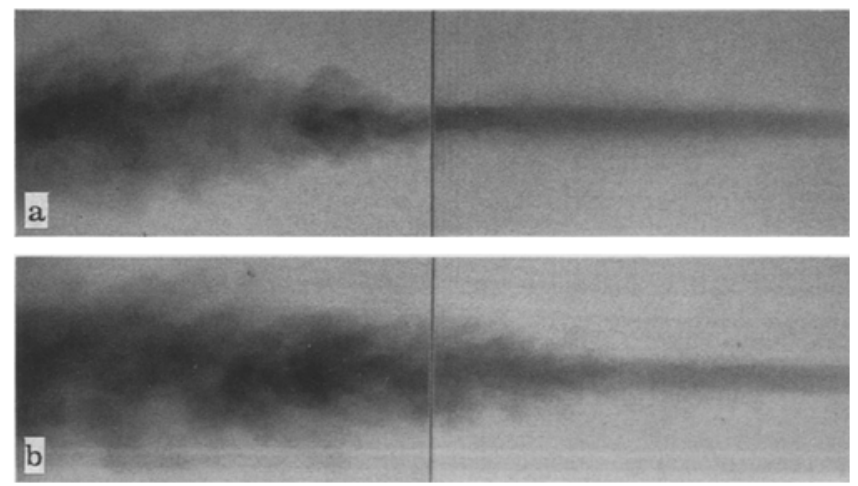

Fig. 6a and b. Integral visualizations of helium jets: a $F=3,800$, $=1,760 ;$ b $F=6,645,=1,760$
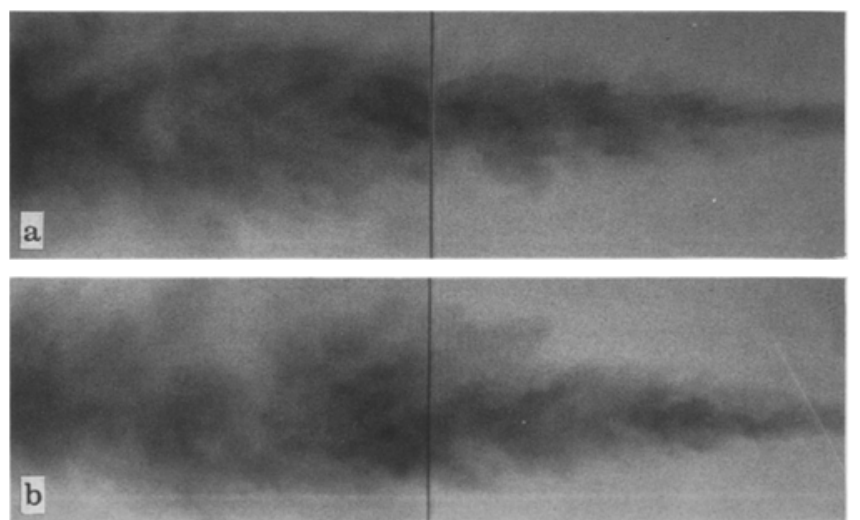

Fig. 7 a and b. Integral visualizations of helium jets: a $F=16,500$, $=2,800 ;$ b $F=37,500,=4,300$

in the momentum-dominated regions being bounded by $z / d=19$ to 190 , respectively. The interferometer viewing area in Figs. 5-7 is about 27 pipe diameters in length. Therefore, the flows in this study can be considered as momentumdominated jets, except in the farthest visible field of the $F=405$ flow.

Using dimensional reasoning, the jet width $\delta(z)$, the mean velocity $U(z)$, and the momentum diameter $d^{*}$ can be shown 
to scale according to:

$U(z) \sim \frac{1}{z}, \quad \delta(z) \sim z, \quad$ and $\quad d^{*}=\frac{2 \dot{m}_{0}}{\left(\pi \varrho_{\infty} J_{0}\right)^{1 / 2}}$

The momentum diameter is the inlet diameter that a jet having the ambient density would require to have the same initial momentum. This length scale allows comparison of experimental results for buoyant jets with those of nonbuoyant jets. In these experiments, the ratio $d / d^{*}$ is about 3 , meaning that the axial length scale corresponds to three times the axial scale for an analogous non-buoyant jet.

Round turbulent jet are among the most studied of turbulent free shear flows; Brown and Roshko's (1974) discovery of large scale vortex-like structures in plane mixing layers led to similar studies of the axisymmetric jet, especially in the near field which is dominated by the effects of the axisymmetric mixing layer. Crow and Champagne (1971) used schlieren visualization to show that the outer edge of this layer adopted a puffing structure; subsequent visualizations by Yule (1978) revealed these to be vortex rings resulting from the roll-up of the vorticity contained in the thin layer between the jet and ambient fluids. Yule showed that these vortex rings were azimuthally unstable and were soon sheared apart. Bruun (1977) used time domain methods to show that vortex interaction produces strong resonances at various locations within the jet's potential core. At a short distance downstream, however, the spectrum becomes similar to that normally associated with isotropic turbulence.

Similarity properties in fully developed turbulent jets of the mean and fluctuating velocity profiles are well-established (e.g., see Wygnanski and Fiedler 1969). Other time-resolved studies of the transport of passive scalar quantities (Chevray and Tutu 1978; Birch et al. 1978; Pitts and Kashiwagi 1985) have demonstrated self-similar behavior of the velocity and scalar fluctuations in the jet far field and have characterized the outer jet region as an intermittently fluctuating interface between turbulent and non-turbulent regions. Relatively few experiments, however, have been conducted to study the large scale motions in the farfield of the jet, that might explain these fluctuations. Tso et al. (1981) attempted to deduce the persistence and size of possible large scale motions in the far-field of a high Reynolds number $(68,000)$ jet through the use of space-time correlations of multiple hot wire signals. Their data indicated that well-correlated vortex motions either persist for long distances downstream or reccur fairly regularly. They further concluded that these motions were well correlated in off-axis regions that were approximately one local jet width in diameter, 3 local jet widths in longitudinal extent, and occupied an azimuthal region of about 90 degrees. Dimotakis et al. (1983) used laser induced fluorescence to visualize the centerline of jet flow seeded with a fluorescing dye. This study provided dramatic evidence that large scale motions play the major role in fluid entrainment, transporting unmixed ambient fluid inward near and sometimes beyond the jet centerline. The study also pointed out that the jet scalar field appears to assume a helical structure, resulting from the helical vortical structure, which in turn may result from the instability of a momentarily isotropic turbulent velocity field. In a subsequent study using the same apparatus, Dahm (1984) made quantitative measurements of both the instantaneous and time-averaged behavior of the scalar concentration along both the center line and a centered, diametrical line in the fully developed region. Among the conclusions of this study were: (1) that the mixing field consisted of large regions spanning one local jet diameter and contained regions of mixed and unmixed fluid; (2) that diametrical scalar concentration field attains a bimodal profile, in which the various flow regions become relatively uniform in concentration prior to mixing by the large scale motions.

The jet images shown in Figs. 5-7 correspond to inlet Reynolds numbers ranging from 1,400 to 4,300 . Proceeding downstream, the jet images darken indicating a streamwise increase in cross-sectional mass of helium $M(z)$ [Eq. (7)]. The total sectional mass, $M(z)$, can be related to average axial velocity $\bar{U}(z)$ by a trivial application of the continuity equation, yielding

$M(z)=\dot{M}_{0} d z / \bar{U}(z)$

where $\dot{M}_{0}$ is the inlet mass flow and $d z$ is the projected detector element width. Since the velocity $U(z)$ scales as $1 / z$, the quantity $M(z)$ should, on the average, grow linearly in $z$. The plot of an instantaneous axial profile of $M(z)$ (Fig. 8) shows that it seems to fluctuate about a linear profile.

Other interesting features of these images include: (1) the sinuous appearance of the scalar concentration field, sug-

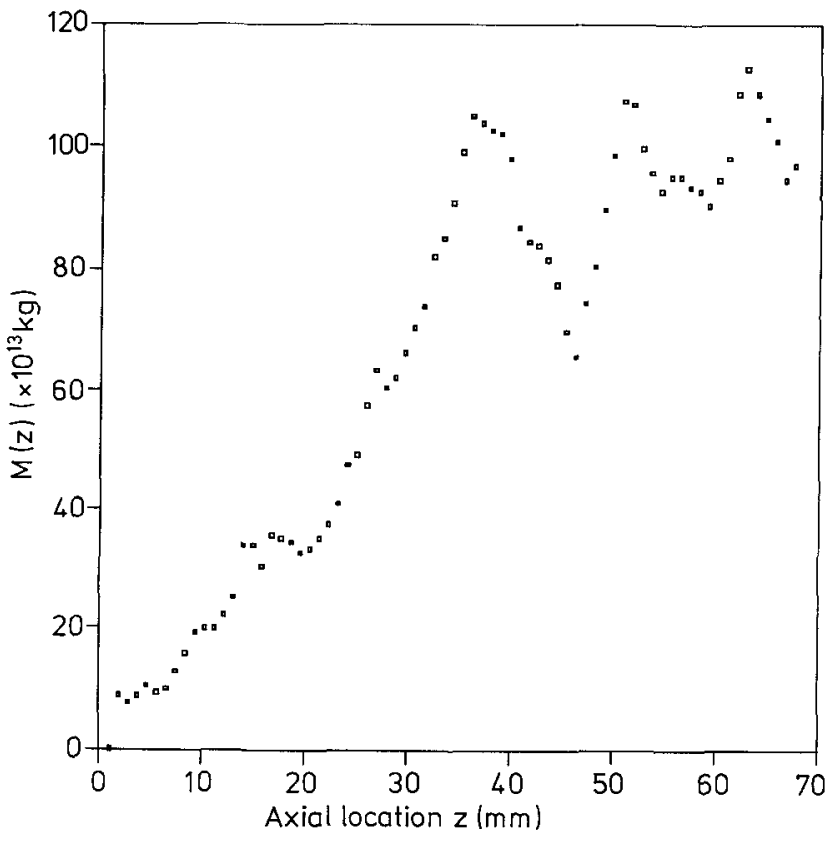

Fig. 8. Instantaneous axial distribution of cross-sectional mass of helium in Fig. $7 \mathrm{~b}$ 

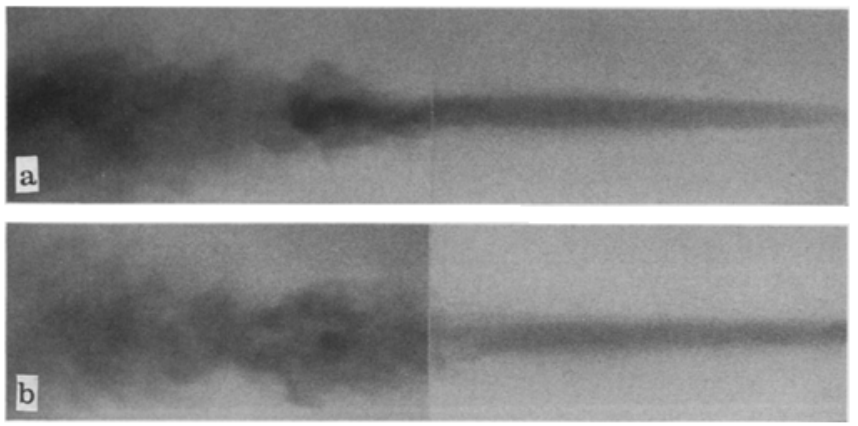

Fig. 9 a and b. Perpendicular integral images of $R e_{0}=1,400$ helium jet

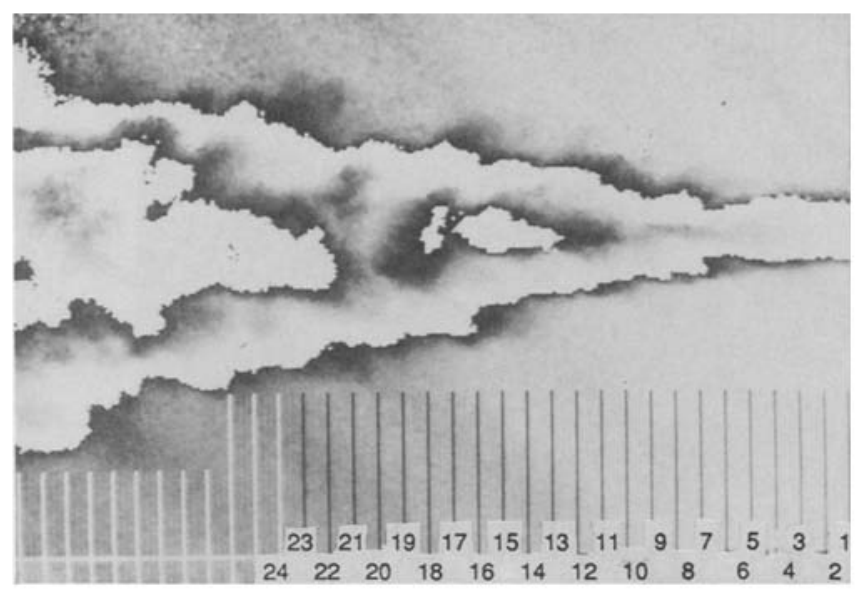

Fig. 10. Perpendicular integral images of $R e_{0}=4,300$ helium jet

gesting a possible helical structure; (2) the locally dark image areas, which suggest the existence of regions of highly concentrated jet fluid; (3) the locally bright image areas well within the jet boundaries, indicating the presence of relatively high concentrations of ambient fluid well within the jet.

The implications of these image features can be further illuminated and, in some cases, contradicted by examining simultaneous perpendicular pairs of these images. Two such pairs are shown in Fig. 9, at a Reynolds number of 1,400, and Fig. 10, at Reynolds number of 4,300. The first image (Fig. 9) illustrates the transition from a laminar to a turbulent jet. By examining the transition region of each image, we see that while the jet narrows in one direction, it becomes wider in the other, implying that the initial region of this jet is elliptical in shape. In the higher Reynolds number jet (Fig. 10) comparison of the two images shows that the apparent regions of high concentration are, in fact, regions of more uniform concentration that have become elongated in the viewing direction. We can also see from these images that the flow appears to undergo a buckling motion in the direction of elongation (arrow no. 1 in Fig. 10). Comparing the orientation of these elongated regions at different axial locations in the flow, one can infer that the scalar field of the jet attains an overall twisted-ribbon structure. The images present further evidence of large-scale motions in the jet, the most

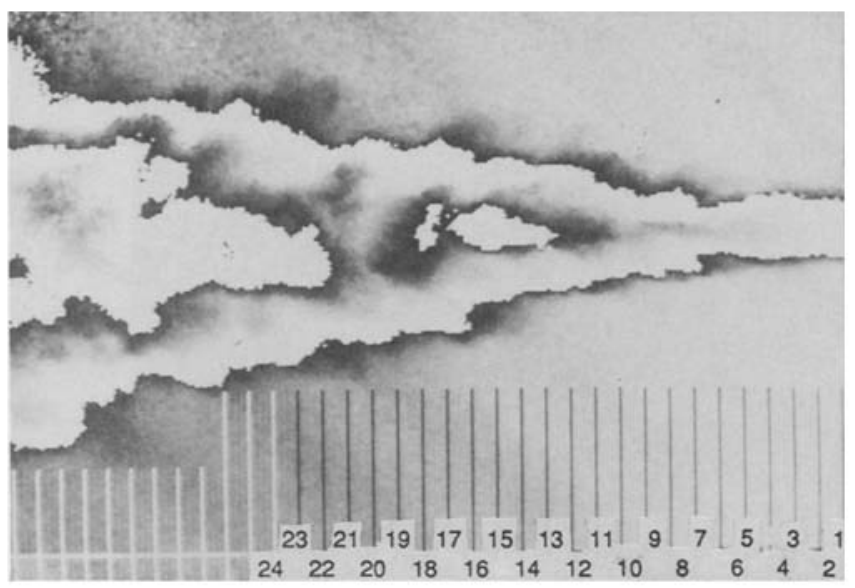

Fig. 11. Sample data image used for tomographic data $(R e=4,300)$

obvious example of which is pointed out by arrow no. 2 in Fig. 10. In this area, a vortical motion appears to have brought nearly unmixed fluid across the jet center line. Examination of the corresponding area of the perpendicular views indicates that this motion is oriented at an angle of about $45^{\circ}$ with the jet center line. Both this motion and the in-plane buckling appear to be well-defined vortical motions whose length scale is roughly equal to the width of the flow.

\section{Tomographic reconstruction of turbulent jets}

Internal visualizations of the jet concentration field by tomographic reconstruction were performed on three turbulent jets, one having a Reynolds number of 2,800 , the other two having a Reynolds number of 4,300. The lower Reynolds number example was reconstructed using data sets of both 25 and 42 views to examine the effects of angular sampling on the reconstruction. The two higher Reynolds number flows were both reconstructed from the same number of views (29) to examine whether there were any obvious image artifacts resulting from the sampling geometry. Figure 11 shows a data image for the jet with $R e=2,800$ and indicates the locations of reconstructed cross-sections.

The reconstruction of image sections 6 and 14 of the jet with $R e=2,800$ demonstrate (1) convergence of the reconstruction algorithm in the presence of noise and (2) the effect of the number of views on the reconstruction quality. These images are shown in Fig. 12. Figure 12a was reconstructed from 42 views while Fig. $12 b$ was reconstructed from 25 views. All four images are degraded by streaks passing through the image, although these are less prominent in the images reconstructed from 42 views. These streak artifacts are well known in medical imaging devices which use backprojection type algorithms (as this system does). Brooks et al. (1979) demonstrated that these artifacts result from the aliasing of the highest spatial frequencies associated with 


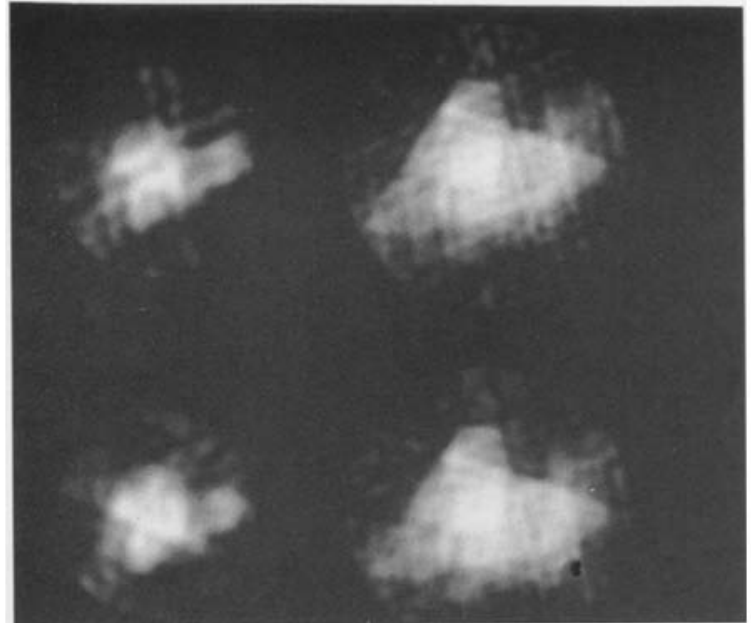

Fig. 12. Comparative reconstructions at axial location 6 and 14 of $R e=2,800$ jet using 42 views and 25 views
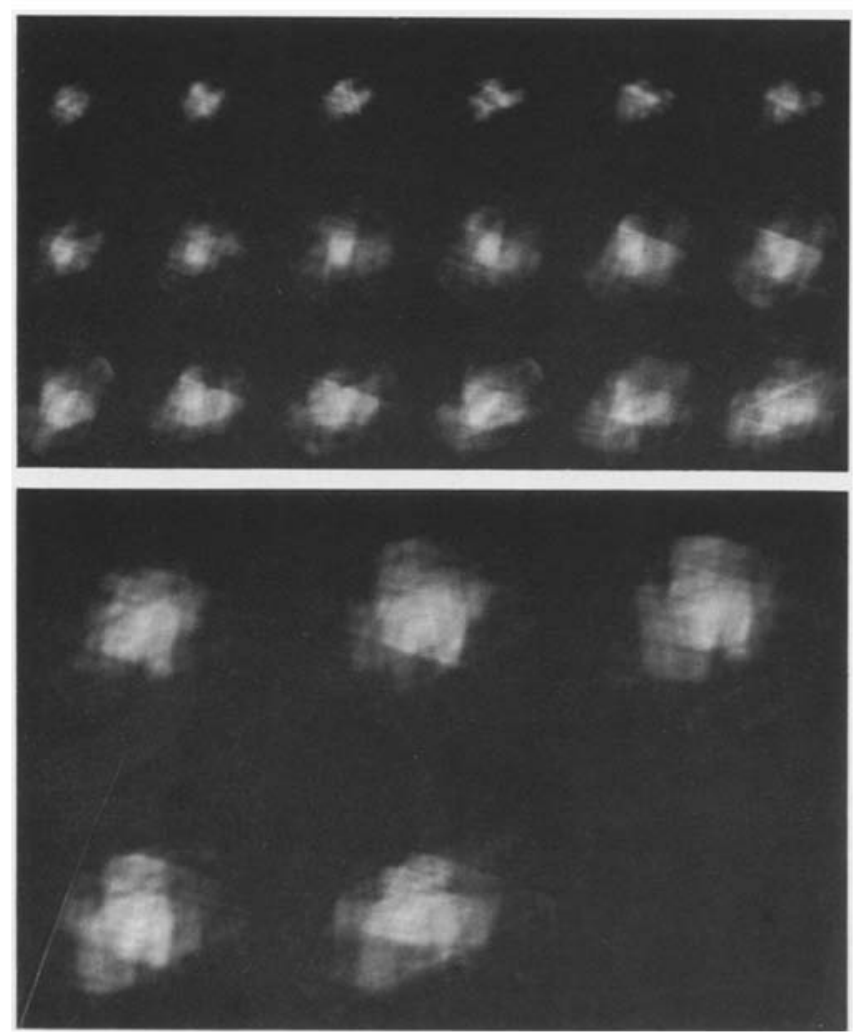

Fig. 13. Reconstructions at axial locations $1-23$ of $R e=2,800$ jet

sharp gradients or edges within the source field. These sharp boundaries cause the term $\partial R(s, \theta) / \partial s$ in the inversion formula [Eq. (3)] to become very large in the presence of a sharp boundary or gradient. If the high frequencies associated with this term are undersampled, the boundary gives rise to a low-frequency, large-amplitude artifact in the backprojection operator. This artifact is then backprojected across the
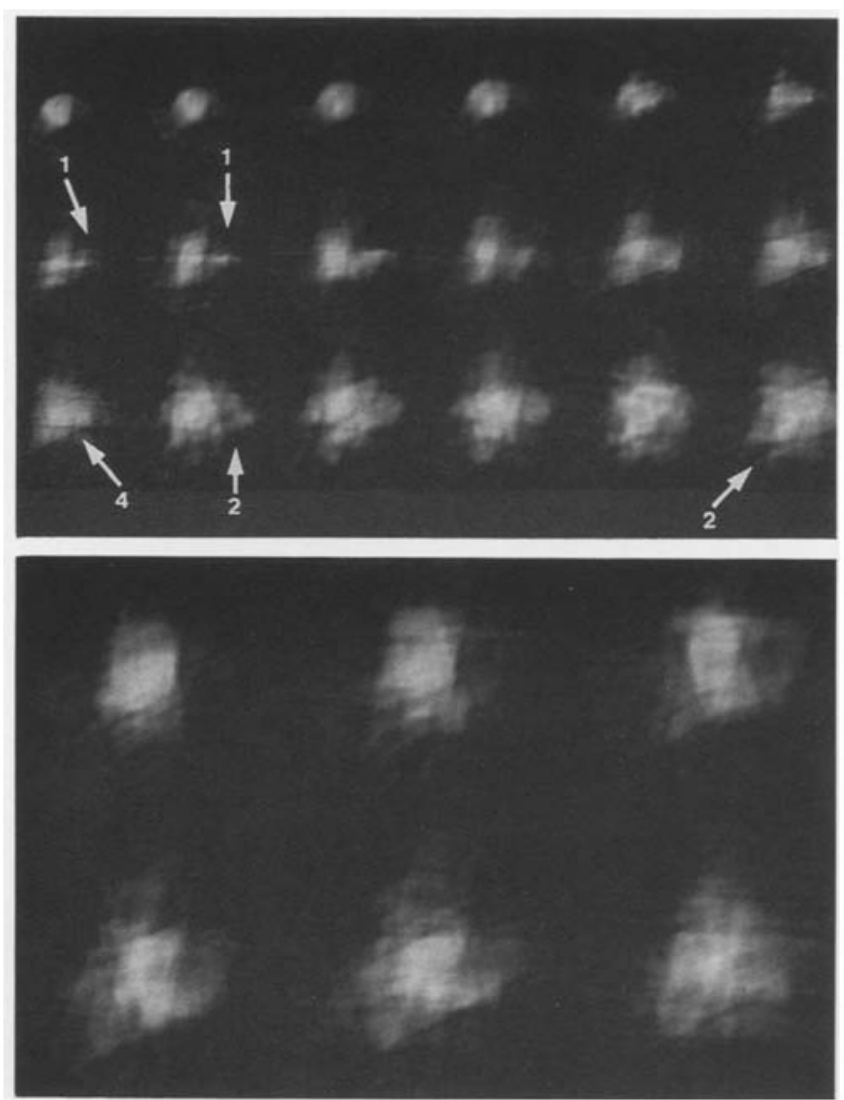

Fig. 14. Reconstructions at axial locations $1-24$ of first $R e=4,300$ jet

image during reconstruction [Eq. (5)], forming a streak which is tangential to the edge that created it.

In spite of the image streaks, the cross-sectional reconstructions do provide insight into the smaller scale structure of the concentration field; indeed, the streaks themselves indicate the presence of high helium concentration gradients in the flow field. Figure 13 shows the instantaneous structure of the first 23 cross-sections of the jet with $R e=2,800$. The first 8 sections show the transition from the parallel flow region through the region of initial instability. Section 2 shows the presence of an apparent azimuthal wave distorting the initially axisymmetric profile. Sections $(3-8)$ in the initial instability region indicate that the flow disturbances have become very irregular. The sections beyond this region show that the cross-section of the jet becomes elongated and appears to twist in a clock-wise direction. At section 19, it has a nearly symmetric circular shape.

The first 24 sections of the two flows with $R e=4,300$ are shown in Figs. 14 and 15. The region near the pipe of the first of these flows (Fig. 14, axial locations 1-3), show the presence of two stream-wise vortex motions entraining ambient fluid toward the jet center. The most probable source of this is a room draft generating a vortex pair. In succeeding downstream flow sections, the bulk of the jet fluid appears to be contained in an elliptical area. Further downstream, the 

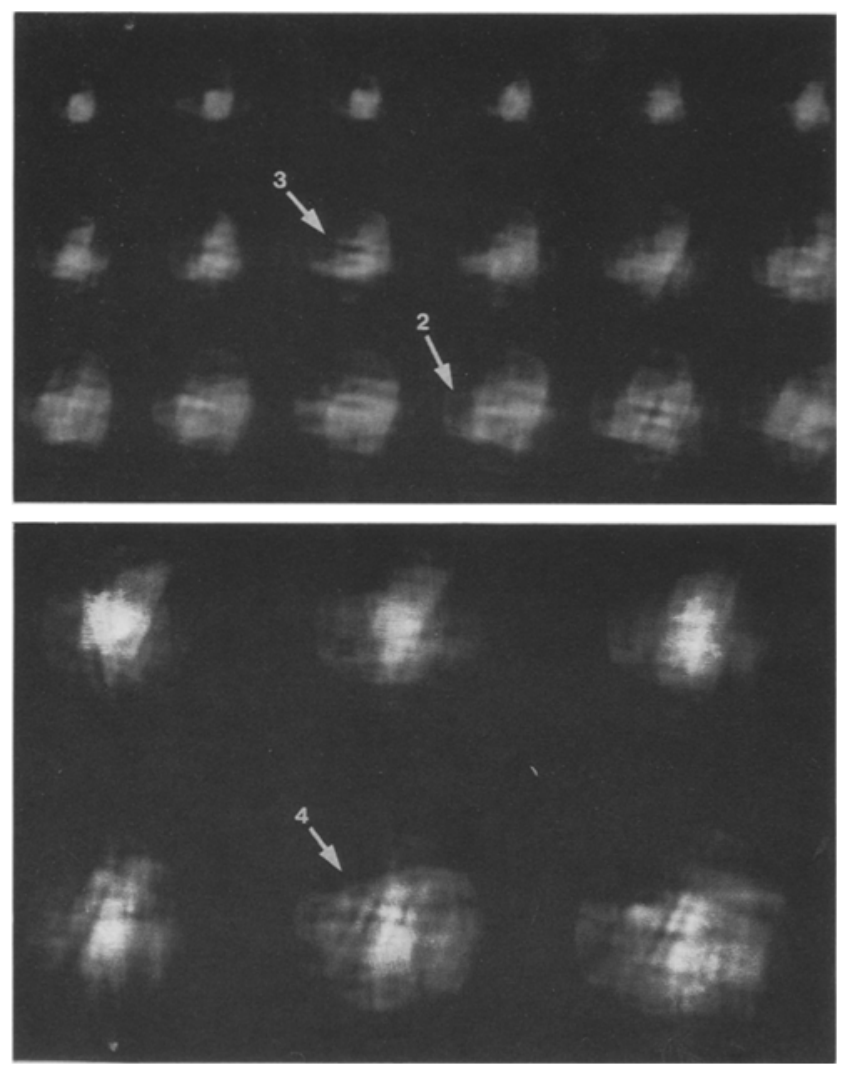

Fig. 15. Reconstructions at axial locations $1-24$ of second $R e=$ 4,300 jet

cross-section is alternatively cross-shaped, round, or elliptical. Similar evolution in the jet cross-section is apparent in the second set of $R e=4,300$ flow images.

In both $R e=4,300$ jets, the cross-sectional images also show the presence of identifiable motions with length scales of half the flow width or less. The first of these is the apparent ejection of fluid away from the jet center region, shown in sections 7 and 8 of Fig. 15 (arrow no. 1). The second of these are apparent vortical enclosures of ambient fluid in the outer regions of the jet (arrow no. 2 in Figs. 14 and 15). These enclosures create sharp interfaces between the concentrated jet fluid and unmixed ambient fluid and can be transported far into the jet (arrow no. 3 in Fig. 15). Steep gradients in these regions create streak artifacts which radiate from these interfaces (arrow no. 4 in Fig. 15).

\section{Discussion and conclusions}

The goal of the jet visualization study was to examine integral and tomographic imaging for the study of turbulent flows. The information in the images presented here does have interesting parallels with the results of other researchers. The integral visualizations tend to confirm the contention of Dimotakis et al. (1983) and Dahm (1984) that turbulent jets are organized into large-scale motions whose

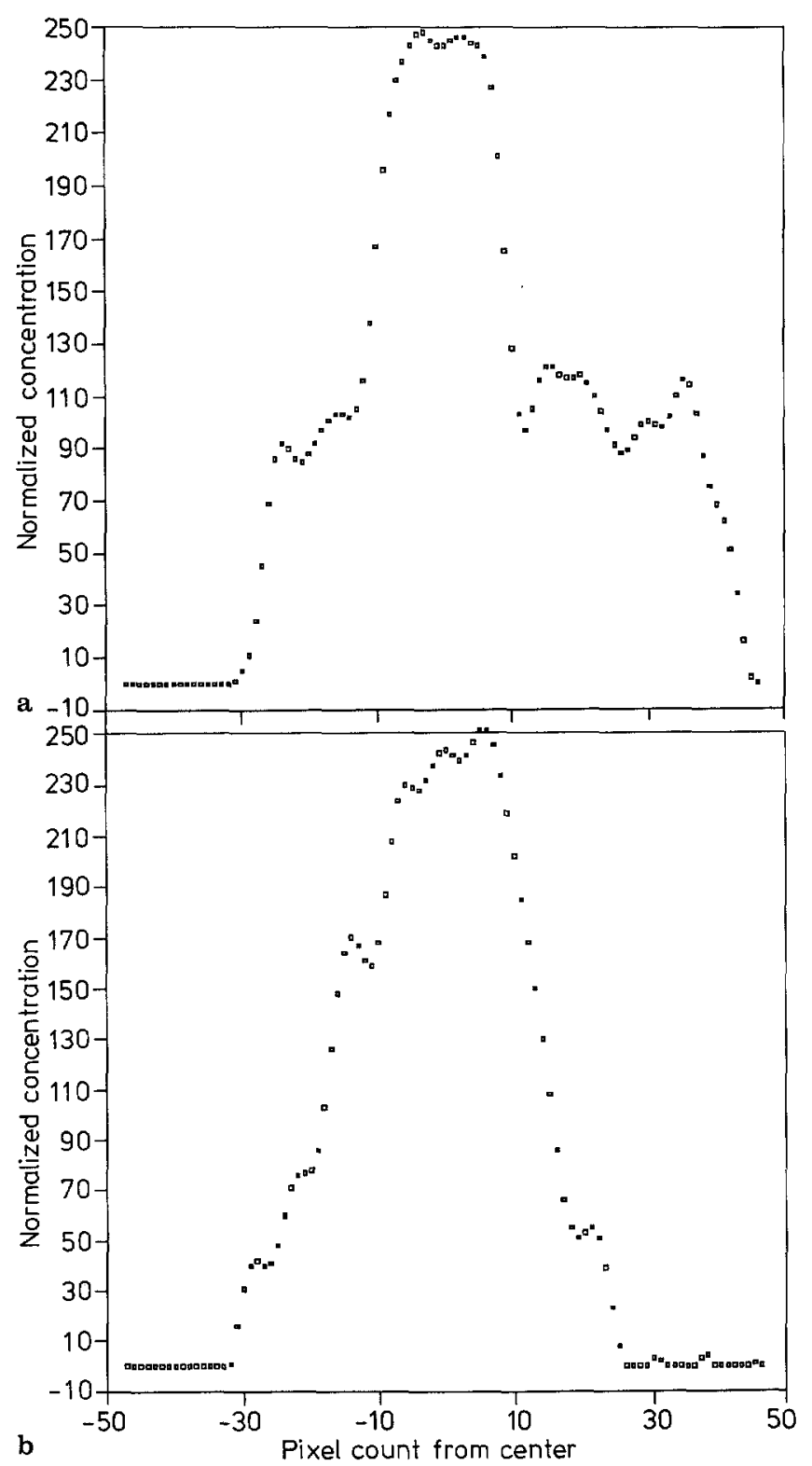

Fig. 16 a and b. Reconstructed image values of $R e=2,800$ jet, axial location 9 along $\mathbf{a} x$-axis and $\mathbf{b} y$-axis

length-scale is of the order of the local flow width. The tomographic reconstructions confirm the presence of nearly unmixed ambient fluid within the jet boundaries and tend to confirm Dahm's results that the jet scalar field is divided into regions of relatively constant concentration. Figure 16 shows the concentration profiles along the $x$ and $y$ axes of axial location 9 of the $R e=2,800$ jet, which demonstrate that the concentration field consists of regions of relatively constant concentration joined by steep concentration gradients.

Although the tomographic image artifacts obscure fine details in the flow and allow only general conclusions about the scalar fields' geometry, the experiments do show that interferometric integral imaging and computed tomography 
can provide insight into complex flow fields. They also indicate the areas of development needed to improve the tomographic images and extend the use of interferometric tomography from visualization to quantitative measurement.

The most obvious need is improvement of the accuracy and spatial resolution of the phase-measuring interferometer. The interferometer's chief error source is the different recording and reconstruction wavelengths; this requires the use of diffuse illumination and closely separated reference waves, causing systematic errors due to overlapping reconstructions and holographic aberrations. These problems would be largely eliminated by using a monochromatic system having continuous-wave and pulsed lasers of the same wavelength or using a rapidly scanning continuous wave laser such as the one employed by Snyder and Hesselink (1988). Alternatively, a system for capturing pulsed laser images on an electronic imaging device during reconstruction would require the use of only one laser. The system accuracy could further be improved by the use of specular illumination sources such as plane waves or diverging waves, which can reduce the interferometer uncertainty to less than $1^{\circ}$ (Cheng and Wyant 1985). Specular illumination would also eliminate fringe localization problems. Multiple specular probe rays can be generated with phase gratings (Radulovich 1977), multiple mirrors (Snyder and Hesselink 1988) or perhaps with fiber optics.

As a final note on the potential for making tomographic measurements in unsteady flows, one must remember that reconstruction accuracy is limited not only by the accuracy of the probe integral measurements, but also by the presence of reconstruction artifacts. These artifacts depend on the suitability of the sampling geometry and the reconstruction algorithm to the source function. Since the reconstructions of turbulent flow fields cannot be verified by independent experiment or analytical solution, the problem of calibration of such instruments and quantitative interpretation of the results is not trivial. This question can probably only be answered by numerical simulation, which will require the development of plausible test objects and will (hopefully) lead to reconstruction algorithms tailored to the properties of turbulent flows.

\section{References}

Bennet, K. E.; Faris, G. W.; Byer, R. L. 1984: Experimental optical fan beam tomography. Appl. Opt. 23, 2678-2685

Birch, A. D.; Brown, D. R.; Dodson, M. G.; Thomas, J. R. 1978: The turbulent concentration field of a methane jet. J. Fluid. Mech. 88 , $431-455$

Breuckmann, B.; Thieme, W. 1985: Computer-aided analysis of holographic interferograms using the phase shift method. Appl. Opt. 24, 2145-2149

Brooks, R. A.; Glover, G. H.; Talbert, A. J.; Eisner, R. L.; DiBianca, F. A. 1979: Aliasing: a source of streaks in computed tomograms. J. Comput. Assist. Tomogr. 3, 511-518

Brown, G. L.; Roshko, A. 1974: On density effects and large structures of turbulent mixing layers. J. Fluid Mech. 64, 475-816
Bruun, H. H. 1977: A time-domain analysis of the large-scale flow structure in a circular jet. Part 1. Moderate Reynolds number. J. Fluid Mech. 88, 641-655

Byer, R. L.; Shepp, L. A. 1979: Two-dimensional remote air-pollution monitoring via tomography. Opt. Lett. 4, 75-77

Chen, C.-J.; Rodi, W. 1980: A review of experimental data of vertical turbulent jets and plumes. Oxford: Pergamon Press

Cheng, Y.-Y.; Wyant, J. C. 1985: Phase shifter calibration in phaseshifting interferometry. Appl. Opt. 24, 3049-3052

Chevray, R. C.; Tutu, K. K. 1978: Intermittency and preferential transport of heat in a round jet. J. Fluid Mech. 88, 133-154

Crow, S. C.; Champagne, F. H. 1971: Orderly structures in jet turbulence. J. Fluid Mech. 48, 541-591

Dändliker, R.; Thalmann, R. 1985: Heterodyne and quasi-heterodyne holographic interferometry. Opt. Eng. 24, 824-831

Dändliker, R.; Moram, E.; Mottier, F. M. 1976: Two-reference beam holographic interferometry. J. Opt. Soc. Am. 60, 23-30

Dändliker, R.; Thalmann, R.; Willemin, J.-F. 1982: Fringe interpolation by two reference beam holographic interferometry. Opt. Commun. 42, 23-29

Dahm, W. J. A. 1984: Experiments on entrainment, mixing and chemical reactions in turbulent jets at large Schmidt number. $\mathrm{Ph}$.D. Thesis, California Institute of Technology, Pasadena/CA

Deans, S. R. 1983: The Radon transform and some of its applications. New York: Wiley

Dimotakis, P. E.; Miake-Lye, R. C.; Papantoniou, D. A. 1983: Structure and dynamics of round, turbulent jets. Phys. Fluids 26, 3185-3192

Faris, G. W.; Byer, R. L. 1986: Quantitative optical tomographic imaging of a supersonic jet. Opt. Lett. 9, 413-415

Hariharan, P. 1985: Quasi-heterodyne holographic interferometry. Opt. Eng. 24, 632-638

Hertz, H. M. 1985: Experimental determination of 2-d flame temperature fields by interferometric tomography. Opt. Commun. $54,131-136$

Hiller, B.; Hanson, R. K. 1988: Simultaneous planar measurements of velocity and pressure fields in gas flows using laser-induced fluorescence. Appl. Opt. 27, 33-48

Kittelson, J. K.; Yu, Y. H. 1985: Transonic rotor flow-measurement technique using holographic interferometry. J. Am. Helicopter. Soc. 30, 3-9

Kreiss, T. 1986: Digital holographic interference-phase measurement using the fourier transform method. J. Opt. Soc. Am. A 3, $897-895$

Leith, E. N.; Upatnieks, J.; Haines, K. A. 1965: Microscopy by wavefront reconstruction. J. Opt. Soc. Am. 55, 981-986

Meier, R. W. 1965: Magnification and third-order aberrations in holography. J. Opt. Soc. Am. 55, 987-992

Pitts, W. M.; Kashiwagi, T. 1985: The application of Rayleigh-light scattering to the study of turbulent mixing. J. Fluid Mech. 141, $391-429$

Radulovich, P. T. 1977: Holographic interferometry of three-dimensional temperature or density fields. Ph.D. Thesis, Department of Mechanical Engineering, The University of Michigan, Ann Arbor/MI

Santoro, R. J.; Semerjian, H. G.; Emmerman, P. J.; Goulard, R. 1981: Optical tomography for flow field diagnostics. Int. J. Heat Mass Transfer 24, 1139-1150

Snyder, R. 1988: Instantaneous three-dimensional optical tomographic measurements of species concentration in a co-flowing jet. Ph.D. Thesis, Department of Aeronautics and Astronautics, Stanford University, Stanford/CA

Snyder, R.; Hesselink, L. 1988: Measurement of mixing fluid flows with optical tomography, Opt. Lett. 13, 87-89

Stuck, B. W. 1977: A new proposal for estimating the spatial concentration of certain types of air pollutants. J. Opt. Soc. Am. 67, $668-678$ 
Sweeney, D. W.; Vest, C. M. 1973: Reconstruction of three-dimensional refractive index fields from multidirectional interferometric data. Appl. Opt. 12, 2649-2664

Tso, J.; Kovasznay, L. S. G.; Hussain, A. K. M. F. 1981: Search for large-scale coherent structures in the nearly self-preserving region of turbulent axisymmetric jet. J. Fluids Eng. 103, 503-508

Vest, C. M. 1979: Holographic interferometry. New York: Wiley

Watt, D. W.; Vest, C. M. 1987: Digital interferometry for flow visualization. Exp. Fluids 5, 401-408

Watt, D. W.; Vest, C. M. 1989: Consistent iterative convolution: A coupled approach to tomographic reconstruction. J. Opt. Soc. A. $6,44-51$
White, F. M. 1974: Viscous flows. New York: McGraw-Hill Wygnanski, I.; Fiedler, H. 1969: Some measurements in the self-preserving jet. J. Fluid Mech. 38, 577-612

Yip, B.; Long, M. B. 1986: Instantaneous planar measurements of the complete three-dimensional scalar gradient in a turbulent jet. Opt. Lett. 11

Yule, A. J. 1978: Large scale structure in the mixing layers of a round jet. J. Fluid Mech. 89, 413-420

Received July 1, 1989 